\title{
Study of Dislocation Boundary Structure in Al-Li Alloy During Bending
}

\author{
Xiao Jin ${ }^{1} \cdot$ Bao-Qin $\mathrm{Fu}^{2} \cdot$ Cheng-Lu Zhang ${ }^{1} \cdot$ Wei Liu ${ }^{1}$
}

Received: 15 April 2015/Revised: 7 July 2015/Published online: 12 August 2015

(c) The Chinese Society for Metals and Springer-Verlag Berlin Heidelberg 2015

\begin{abstract}
The dislocation boundary structures of 2060-T8 alloy during bending were investigated by backscattered electron imaging, electron backscattered diffraction, and misorientation axes maps. Experimental result shows that typical dislocation boundary structures, which depend on grains' orientation, are formed in grains during bending. The microstructure of type A is mainly observed in grains near brass, copper, and Goss orientations; microstructure of type B is mainly found in grains near S orientation; microstructure of type $\mathrm{C}$ is mainly seen in grains near Cube orientation. The angle between geometrically necessary boundaries (GNBs) and force axis is in the range of $-45^{\circ}$ to $-30^{\circ}$ and $30^{\circ}$ to $45^{\circ}$. Most of the GNBs are approximately parallel to the trace of $\{111\}$ slip planes which are identified by Schmid factor analysis.
\end{abstract}

KEY WORDS: Al-Li alloy; Plastic deformation; Electron backscattering diffraction (EBSD); Microstructure

\section{Introduction}

The Al-Li alloys have been widely used for aerospace structures due to its excellent properties of high strength and low density $[1,2]$. The 2060 alloy is one of the third-generation Al-Li products developed by Alcan Inc., in 2011 for the manufacture of fuselage and lower wing skin structure in replacement of traditional aluminum alloys [3]. For 2060 alloy, the process of obtaining appropriate size and shape is the most important step for working as aircraft structural

Available online at http://link.springer.com/journal/40195

Wei Liu

liuw@mail.tsinghua.edu.cn

1 Laboratory of Advanced Materials, School of Material Science and Engineering, Tsinghua University, Beijing 100084, People's Republic of China

2 Key Laboratory for Radiation Physics and Technology, Institute of Nuclear Science and Technology, Sichuan University, Chengdu 610065, People's Republic of China component, and one commonly used deformation processing is bending, which is an environment-friendly and lowwaste method in the manufacture of structural parts [4].

It is regarded that grains are subdivided into cell blocks (CBs) containing ordinary dislocation cells during plastic deformation in FCC metals with high stacking fault energy. The cell blocks are defined by extended planar boundaries referred to as geometrically necessary boundaries (GNBs), while the dislocation cells inside the cell blocks are defined by boundaries referred to as incidental dislocation boundaries (IDBs) [5-7]. The mechanical properties of material can be drastically affected by the structure parameters of dislocation boundary $[6,8,9]$; hence, it is important to investigate the microstructure evolution during bending of this advanced industrial materials.

In the past decades, many studies have been done to investigate the microstructure and dislocation boundaries during rolling, and the experimental results show that the types of microstructure and dislocation boundaries are depended on the grains' orientation during rolling in $\mathrm{Al}$, 
and the three different types of microstructures correspond to the three different areas in the inverse pole figure in $\mathrm{Al}$ and $\mathrm{Cu}$ during tension $[6,10,11]$. It is generally accepted that different active slip systems can be triggered in grains with different orientations, which can lead to the different microstructure formation [12, 13]. The deformation microstructure depended on the grain's orientation has been verified in pure single-crystal and polycrystalline metals during rolling, tension, and compression [10, 1418]. However, there is still a lack of studies on the evolution of the dislocation boundary structure of alloy during bending.

Electron backscattered diffraction (EBSD) is one of the effective methods to characterize the microstructure in grains, especially in grains under deformation with large area. In the present work, EBSD and backscattered electron (BSE) imaging techniques are combined to characterize the microstructure in specimens; thus, the statistical result of microstructure and orientation information within grains is accurately collected.

\section{Experimental}

In the present work, the material used was a 2060 aluminum alloy commercially made by Alcoa Inc., and the heat treatment was T8. T8 heat treatment is identified as: solution heat treatment at a temperature range of 499-507 ${ }^{\circ} \mathrm{C}$ in accordance with AMS2722, during which the grains near the surfaces are recrystallized; artificial aging has been performed at $(143 \pm 2.8){ }^{\circ} \mathrm{C}$ for $35 \mathrm{~h}$.

The plates were bent by cold-rolled forming, and their bend radii were 10,5 , and $3 \mathrm{~mm}$, respectively, and more than eight specimens were investigated under every bend radius. The bending axe is along the transverse direction (TD), and the strain under different bend radii at study regions was calculated by finite element (FE) simulation. Split specimens of $10 \mathrm{~mm} \times 3 \mathrm{~mm} \times 2 \mathrm{~mm}$ [along the rolling direction (RD), transverse direction (TD), and normal direction (ND), respectively] were cut by linear cutting machine from the unbent and bent plates. In bent specimen, region near outer surface is under tension and region near inner surface is under compression. The strain was decreasing from surface to neutral layer in bent specimen. The regions near the surface were studied using EBSD and BSE.

Specimens for characterization, whose upper surface was $\mathrm{RD} \times \mathrm{ND}$, were first mechanically ground and then electropolished in $\mathrm{HClO}_{4} / \mathrm{C}_{2} \mathrm{H}_{5} \mathrm{OH}$ (10:90, in volume fraction) solution at room temperature under applied voltage of $20 \mathrm{~V}$ for $15-20 \mathrm{~s}$.

The EBSD data were collected by Oxford Nordly 2, with the accelerating voltage of $20 \mathrm{kV}$, magnification of
2000, the scanned area of $100 \mu \mathrm{m} \times 100 \mu \mathrm{m}$, working distance of $15 \mathrm{~mm}, 70^{\circ}$ tilt angle, to the EBSD probe scan over the same area of SEM scan, and step size was $0.1 \mu \mathrm{m}$. The texture was analyzed by $h k l$ Channel 5 EBSD analysis system (Oxford Instruments, Oxford, England).

For each of the BSE image, an EBSD map was taken at the same region, in order to identify the orientations of the grains seen in the BSE image. In the current work, a software package was designed to characterize the microstructure of grains' subdivision during deformation with misorientation axes. All the EBSD maps were restructured. The procedure for calculating such misorientation axes maps was described in the literature $[11,16$, 19].

\section{Results and Discussion}

\subsection{Misorientation Axes Maps}

It is reported that GNBs cannot be characterized by commercial EBSD software especially under small strain; however, previous researches found that the microstructure characterized by misorientation axes maps well, and more microstructure can be observed under deformation [19-21].

In EBSD maps, every grain is constituted by different numbers of pixels, and each pixel has different orientation information. Misorientation axes maps in each grain are analyzed as an independent unit, and the average orientation is calculated. The misorientation of each pixel relative to grain's average orientation can be represented as an angle-axis pair $(\theta / r)$ and reconstruct the misorientation axes.

In this angle-axis pair, the axis orientation is unit vector which can be represented by the coordinates $(x, y, z)$ in sample coordinate, and the axis orientation corresponds to one point in the surface of unit sphere. Every point in the surface has its different color. For example, the color corresponding to the end point $(1,0,0)$ in the positive $x$ axis is $R=255, G=128, B=128$; the color in the opposite point $(-1,0,0)$ is $R=1, G=128, B=128$.

In misorientation axes maps, the misorientation of each pixel to grain's average orientation can be represented by different colors; hence, the rotated axes' spatial orientation distribution of each pixel can be characterized.

\subsection{Microstructure of 2060 Alloy in Different Bend Radii}

Figure 1 shows the microstructure of 2060-T8 when the bend radius reaches $10 \mathrm{~mm}$, and the average strain is nearly 0.1. Figure 1a is the BSE image which shows a typical deformation microstructure that band structures are formed 

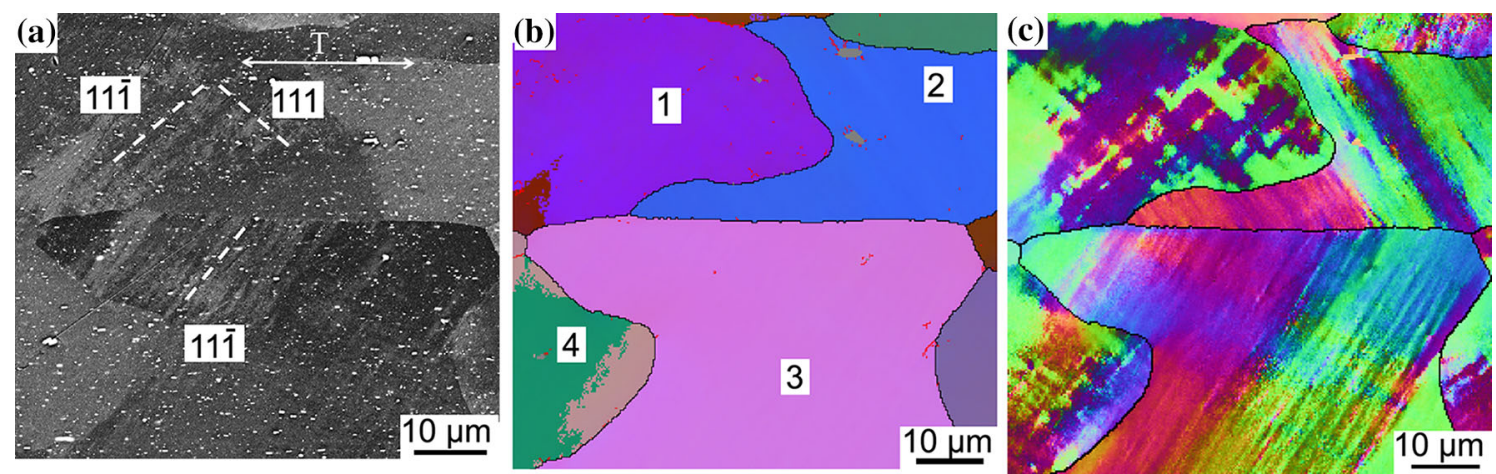

Fig. 1 Microstructure of 2060-T8 alloy with the bend radius is $10 \mathrm{~mm}$ (black lines represent grain boundary with the misorientation larger than $\left.15^{\circ}\right)$ : a BSE image; $\mathbf{b}$ EBSD map reconstructed from all Euler; $\mathbf{c}$ misorientation axes map

in some grains, and these band structures are the arrangement of the dislocation boundaries [7]. Grain 1 and grain 3 are subdivided into several smaller regions, and cell-blocktype microstructure appears. With the application of trace line methods, two sets of GNBs can be observed in grain 1 (near brass orientation, $\{110\}\langle 112\rangle$ ), and only one set of GNBs can be observed in grain 3 ( $\mathrm{S}$ orientation, $\{123\}\langle 63-4\rangle$ ), which are parallel with the traces of $\{111\}$ slip planes. The result shows the GNBs with a certain angle to the force axis (FA). In Fig. 1b of EBSD reconstructed map, only a few low-angle grain boundaries (LAGBs, $2^{\circ}-$ $10^{\circ}$, the red lines) can be found in the same grains. It means that the EBSD reconstructed map cannot characterize dislocation boundary structures under this deformation. Figure 1c is the misorientation axes map, and the similar microstructure can be found obviously in grain 1 and grain 3 . Some less visible structures can be observed in grain 2 and grain 4 (Cube orientation, $\{001\}\langle 100\rangle$ ) due to small strain

Figure 2 shows the microstructure of 2060-T8 when the bend radius reaches $5 \mathrm{~mm}$, and average strain is nearly 0.12 . Figure $2 \mathrm{a}$ and $\mathrm{b}$ shows the microstructure under tension. In grain 2 (copper orientation, $\{112\}\langle 11-1\rangle$ ), one set of GNBs can be observed obviously in Fig. 2a, while the other set of GNBs can hardly be observed. Compared to the BSE image, Fig. $2 b$ reveals a better contrast deformation microstructure in grain 2, with two sets of GNBs shown clearly in misorientation axes map. In grain 4 (brass orientation), two sets of GNBs were observed in Fig. 2a, b. They form angles of $-42^{\circ}$ and $34^{\circ}$ to the tangent of tension (positive values are defined as being anticlockwise to the tension for a rotation axis out of the plane of the paper). Only one set of GNBs is observed in grain 6 (near S orientation). And grain 1 (Cube orientation) and grain 3 in Fig. 2a, b show no boundaries are formed.

Figure $2 \mathrm{c}, \mathrm{d}$ shows the microstructure under compression. Two sets of GNBs can be seen in grain 4 (brass orientation). In grain 1 (S orientation) and in grain 6 , only one set of GNBs can be seen. The GNB structures cannot be observed in grain 2 and grain 3 (Cube orientation). In grain 5, the BSE image shows little microstructure information, but the misorientation axes map shows the two sets of GNB microstructures clearly. The trace line method shows one of the GNBs in grain 4 which are parallel with the traces of $\{111\}$ slip planes, while the other set of GNBs are not. The angle between the GNBs and traces of $\{111\}$ is nearly $15^{\circ}$. The GNBs in other grains are nearly parallel with the traces of $\{111\}$ slip planes.
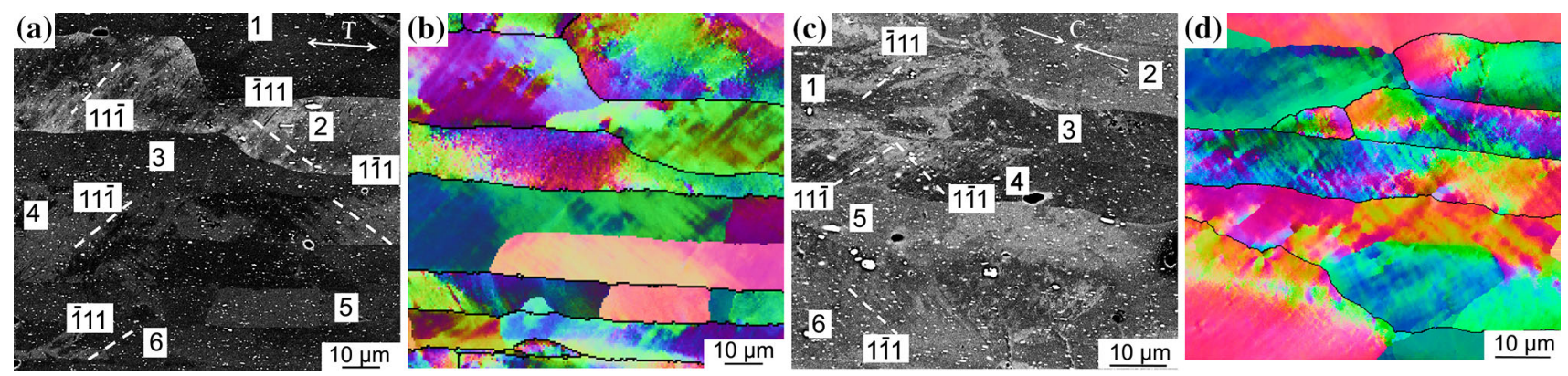

Fig. 2 Microstructure of 2060-T8 alloy with the bend radius is $5 \mathrm{~mm}$ : a BSE image under tension; $\mathbf{b}$ misorientation axes map under tension; c BSE image under compression; $\mathbf{d}$ misorientation axes map under compression 

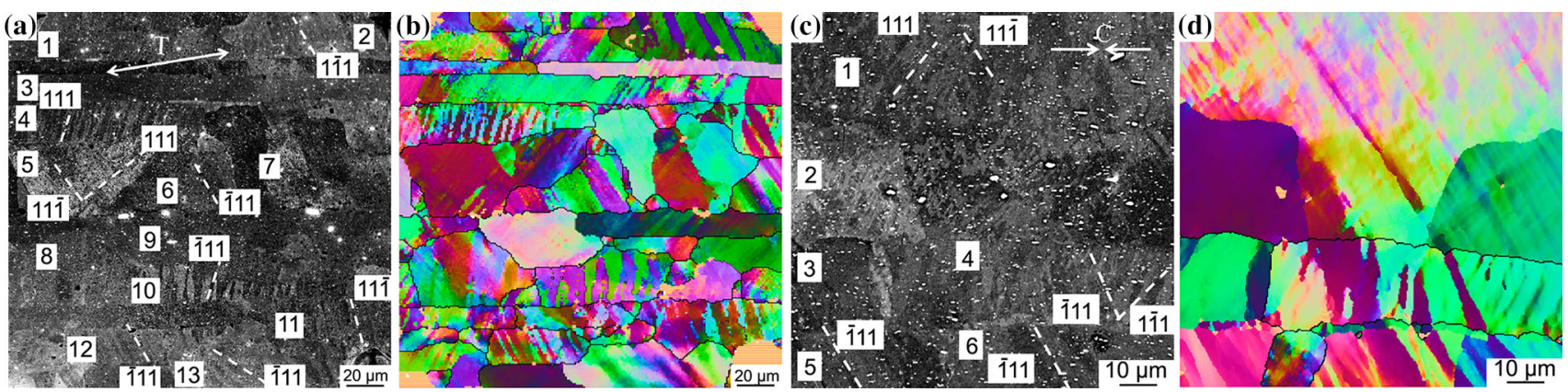

Fig. 3 Microstructure of 2060-T8 alloy with the bend radius is $3 \mathrm{~mm}$ : a BSE image under tension; b misorientation axes map under tension; c BSE image under compression; $\mathbf{d}$ misorientation axes map under compression

Figure 3 shows the microstructure of 2060-T8 when the bend radius reaches $3 \mathrm{~mm}$, and the strain in the observed area is near 0.15 .

Figure $3 \mathrm{a}, \mathrm{b}$ shows the microstructure under tension. From BSE image, it is found that the subdivided parts are formed in grain 4, grain 10, and grain 11 obviously in BSE images. These parts are also found in aluminum alloys with medium strain under cold rolling, and these are development of GNBs in grains with larger misorientation [20]. In some grains, two sets of GNBs are seen. For example, in grain 5 (brass orientation), two sets of GNBs with angles of $45^{\circ}$ and $-31^{\circ}$ to the FA are observed. The trace analysis shows that these two GNBs are close to the traces of the

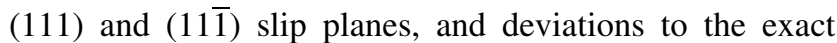
trace angles are $<5^{\circ}$. In grains $2,4,6,10,11,12$, and 13 , only one set of GNBs is formed, and most of these grains are $\mathrm{S}$ orientations except grain 10 which is in brass orientation. Some GNBs are well developed, and some are not seen obviously. Most of the GNBs are nearly parallel to the trace of $\{111\}$ slip planes with small deviation except the GNBs in grain 10 with $-30^{\circ}$ to the (111) trace of slip plane and $-55^{\circ}$ to the FA. (a)

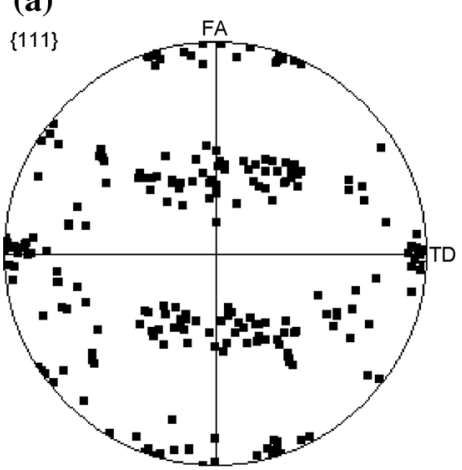

(b)

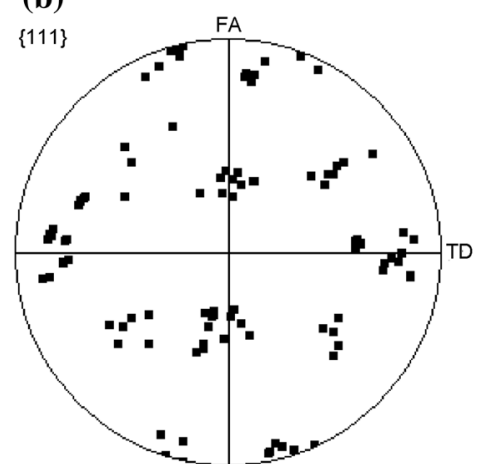

(c)

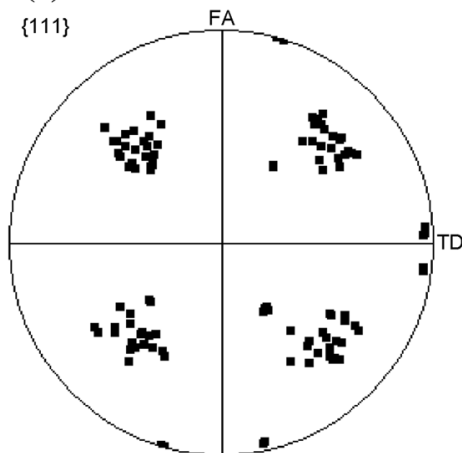

Fig. $4\{111\}$ pole figures of the grains with different microstructures: a type A, two sets of GNBs; $\mathbf{b}$ type B, one set of GNBs; $\mathbf{c}$ type C, large cell structure
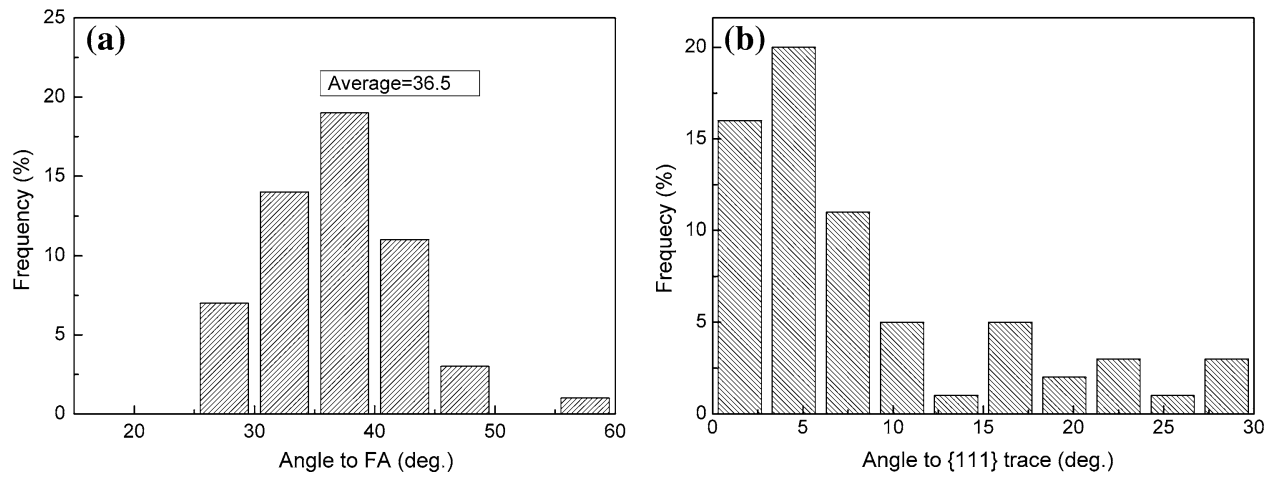

Fig. 5 Angles between GNBs and FA in grains 
Table 1 Schmid factors of all slip systems in grains during bending

\begin{tabular}{|c|c|c|c|c|c|c|c|c|c|c|c|c|c|c|}
\hline \multirow[t]{2}{*}{ Figures } & \multirow[t]{2}{*}{ Grain } & \multirow{2}{*}{$\begin{array}{l}\text { Plane } \\
\text { Direction } \\
\text { Slip system }\end{array}$} & \multicolumn{3}{|l|}{111} & \multicolumn{3}{|l|}{$\overline{111}$} & \multicolumn{3}{|l|}{$\overline{1} 11$} & \multicolumn{3}{|l|}{$1 \overline{1} 1$} \\
\hline & & & $\begin{array}{l}01 \overline{1} \\
a 1\end{array}$ & $\begin{array}{l}\overline{1} 01 \\
a 2\end{array}$ & $\begin{array}{l}1 \overline{1} 0 \\
a 3\end{array}$ & $\begin{array}{l}0 \overline{11} \\
b 1\end{array}$ & $\begin{array}{l}101 \\
b 2\end{array}$ & $\begin{array}{l}\overline{1} 10 \\
b 3\end{array}$ & $\begin{array}{l}01 \overline{1} \\
c 1\end{array}$ & $\begin{array}{l}101 \\
c 2\end{array}$ & $\begin{array}{l}1 \overline{1} 0 \\
c 3\end{array}$ & $\begin{array}{l}0 \overline{11} \\
d 1\end{array}$ & $\begin{array}{l}\overline{1} 01 \\
d 2\end{array}$ & $\begin{array}{l}110 \\
d 3\end{array}$ \\
\hline \multirow[t]{4}{*}{ Figure 1a } & 1 & & 0.43 & 0.43 & -0.39 & 0.38 & -0.42 & -0.41 & 0.38 & 0.01 & -0.19 & -0.04 & -0.21 & 0.13 \\
\hline & 2 & & 0.18 & -0.27 & 0.09 & -0.17 & -0.12 & 0.29 & -0.37 & -0.07 & 0.44 & -0.01 & 0.08 & -0.06 \\
\hline & 3 & & -0.02 & 0.11 & -0.09 & -0.38 & -0.05 & 0.43 & -0.25 & -0.09 & 0.31 & 0.17 & 0.21 & 0.08 \\
\hline & 4 & & -0.04 & 0.37 & 0.04 & 0.11 & 0.26 & -0.17 & -0.14 & -0.30 & -0.11 & 0.26 & 0.41 & -0.22 \\
\hline \multirow[t]{6}{*}{ Figure 2a } & 1 & & 0.02 & 0.04 & -0.04 & -0.27 & -0.11 & 0.39 & 0.01 & -0.24 & 0.29 & 0.27 & 0.11 & -0.40 \\
\hline & 2 & & 0.23 & -0.06 & -0.36 & 0.43 & 0.00 & -0.43 & 0.37 & 0.00 & -0.39 & 0.31 & 0.05 & -0.23 \\
\hline & 3 & & -0.01 & -0.06 & 0.07 & -0.18 & -0.15 & 0.33 & 0.15 & -0.38 & 0.24 & 0.32 & -0.48 & 0.16 \\
\hline & 4 & & 0.42 & -0.03 & -0.38 & 0.41 & 0.01 & -0.43 & 0.40 & -0.01 & -0.38 & 0.40 & 0.03 & -0.43 \\
\hline & 5 & & -0.04 & 0.02 & 0.03 & 0.11 & 0.30 & -0.42 & 0.37 & -0.26 & -0.12 & 0.22 & 0.04 & -0.27 \\
\hline & 6 & & 0.00 & -0.36 & 0.36 & -0.07 & -0.37 & 0.44 & 0.00 & -0.43 & 0.43 & 0.07 & -0.44 & 0.37 \\
\hline \multirow[t]{6}{*}{ Figure $2 \mathrm{c}$} & 1 & & 0.12 & 0.00 & -0.12 & 0.36 & 0.23 & -0.43 & 0.41 & -0.24 & -0.39 & 0.27 & -0.01 & -0.35 \\
\hline & 2 & & 0.13 & -0.46 & 0.32 & 0.01 & -0.43 & 0.42 & -0.13 & -0.32 & 0.45 & 0.00 & -0.30 & 0.30 \\
\hline & 3 & & 0.02 & -0.43 & 0.11 & -0.28 & 0.48 & -0.20 & -0.12 & 0.41 & -0.29 & 0.18 & -0.37 & 0.18 \\
\hline & 4 & & 0.03 & 0.01 & -0.04 & 0.14 & 0.47 & -0.33 & -0.19 & -0.14 & -0.15 & -0.12 & -0.32 & 0.36 \\
\hline & 5 & & 0.24 & 0.06 & -0.30 & 0.26 & 0.11 & -0.36 & 0.49 & -0.18 & -0.31 & 0.48 & -0.13 & -0.35 \\
\hline & 6 & & 0.36 & -0.22 & -0.14 & 0.24 & 0.09 & -0.33 & 0.02 & 0.00 & -0.02 & 0.14 & 0.31 & -0.45 \\
\hline \multirow[t]{13}{*}{ Figure $3 \mathrm{a}$} & 1 & & 0.22 & -0.05 & -0.16 & 0.25 & 0.25 & -0.50 & 0.31 & -0.12 & -0.19 & 0.28 & 0.19 & -0.47 \\
\hline & 2 & & -0.01 & -0.09 & 0.09 & -0.22 & -0.18 & 0.19 & 0.05 & -0.36 & 0.31 & 0.27 & -0.42 & 0.40 \\
\hline & 3 & & 0.01 & -0.08 & 0.07 & -0.30 & -0.17 & 0.48 & -0.13 & -0.26 & 0.39 & 0.19 & -0.35 & 0.16 \\
\hline & 4 & & 0.32 & -0.18 & -0.14 & 0.22 & 0.16 & -0.38 & 0.05 & -0.01 & -0.04 & 0.15 & 0.33 & -0.48 \\
\hline & 5 & & 0.44 & -0.04 & -0.41 & 0.44 & -0.03 & -0.41 & -0.37 & 0.03 & -0.40 & -0.24 & 0.03 & -0.33 \\
\hline & 6 & & 0.22 & -0.34 & 0.12 & -0.10 & -0.21 & 0.31 & -0.32 & -0.12 & 0.45 & 0.00 & 0.01 & -0.01 \\
\hline & 7 & & 0.32 & -0.06 & -0.27 & 0.33 & 0.15 & -0.49 & 0.38 & -0.10 & -0.28 & 0.36 & 0.11 & -0.48 \\
\hline & 8 & & 0.18 & -0.22 & 0.05 & -0.17 & -0.02 & 0.19 & -0.38 & -0.01 & 0.39 & -0.04 & 0.20 & -0.16 \\
\hline & 9 & & 0.06 & -0.16 & 0.10 & -0.31 & -0.16 & 0.47 & -0.31 & -0.16 & 0.47 & 0.06 & -0.16 & 0.10 \\
\hline & 10 & & 0.16 & -0.37 & 0.21 & -0.12 & -0.32 & 0.49 & -0.25 & -0.24 & 0.44 & 0.03 & -0.19 & 0.16 \\
\hline & 11 & & 0.01 & 0.11 & -0.11 & -0.38 & -0.08 & 0.36 & 0.01 & -0.18 & 0.17 & 0.27 & 0.08 & -0.37 \\
\hline & 12 & & 0.12 & -0.19 & 0.12 & -0.14 & -0.32 & 0.38 & -0.34 & -0.12 & 0.44 & 0.00 & 0.02 & -0.01 \\
\hline & 13 & & 0.06 & -0.10 & 0.04 & -0.33 & 0.00 & 0.34 & -0.43 & 0.00 & 0.43 & -0.03 & 0.09 & -0.05 \\
\hline \multirow[t]{6}{*}{ Figure $3 c$} & 1 & & 0.17 & -0.44 & 0.47 & -0.03 & -0.40 & 0.42 & -0.19 & -0.28 & 0.27 & 0.01 & -0.24 & 0.23 \\
\hline & 2 & & -0.04 & -0.35 & 0.40 & -0.05 & -0.35 & 0.40 & 0.06 & 0.39 & -0.45 & 0.06 & -0.39 & 0.45 \\
\hline & 3 & & 0.03 & -0.07 & 0.04 & -0.37 & -0.07 & 0.44 & -0.37 & -0.07 & 0.44 & 0.03 & -0.06 & 0.03 \\
\hline & 4 & & 0.11 & 0.05 & -0.16 & 0.14 & 0.10 & -0.24 & 0.49 & -0.30 & -0.19 & 0.46 & -0.25 & -0.21 \\
\hline & 5 & & -0.07 & -0.02 & 0.08 & 0.09 & 0.18 & -0.11 & 0.44 & -0.37 & -0.07 & 0.29 & -0.17 & -0.27 \\
\hline & 6 & & -0.06 & 0.01 & 0.05 & 0.22 & -0.43 & -0.28 & 0.09 & 0.34 & -0.36 & 0.16 & 0.11 & -0.19 \\
\hline
\end{tabular}

Figure $3 \mathrm{c}$ and $\mathrm{d}$ shows the microstructure under compression. Grain 1 (copper orientation) shows that two sets of GNBs are nearly parallel to the traces of $\{111\}$ slip plane. Large equiaxial cells in grain 2 (Cube orientation, $\{001\}\langle 110\rangle$ ) and these microstructure are similar to that observed in the Cube single crystals after plane strain compression [21]. In grain 4, one set of GNBs is aligned to the trace $(11 \overline{1})$ plane, and the other set of GNBs deviates from the (111) slip plane trace by $20^{\circ}$. One set of GNBs can be seen in grain 5 ( $\mathrm{S}$ orientation) and grain 6 ( $\mathrm{S}$ orientation), and all of them are nearly parallel to the trace of (111) slip plane.

\subsection{Effect of Grain Orientation on Microstructure}

According to the preceding studies, it is found that the formation of the typical microstructure depends on the grain orientation during plastic deformation [22, 23]. The 
microstructure can be classified into three types based on the observed subdivision and dislocation boundary alignment as in previous studies [7, 20]: Type A grains contain two sets of GNBs; type B grains contain one set of GNBs; type $\mathrm{C}$ grains primarily contain large cells.

$\{111\}$ pole figures of grains with different microstructures are shown in Fig. 4. More than 130 grains are studied, and grains containing typical microstructure are counted. The average orientation of every grains is collected and put into Channel 5 to make $\{111\}$ pole figures. The statistical results are similar to previous studies [7]. In Fig. 4, grains primarily near brass, copper, and Goss orientations form into type A microstructure. These phenomena exist in other materials, such as $\mathrm{Ni}, \mathrm{Al}$, the type A microstructure formed during cold deformation [24-26]. Most of these grains near the $\mathrm{S}$ orientation form into type $\mathrm{B}$ microstructure, and grains near Cube orientation form into type $\mathrm{C}$ structure, but not all of the grains follow the rules strictly. Grain 2 in Fig. 2a with Cube orientation shows type A microstructure, and this phenomenon has been reported in single $\mathrm{Al}$ crystal with Cube orientation during cold deformation [21].

\subsection{Analysis of GNBs and Schmid Factors}

Although many works have been done to investigate whether GNBs have macroscopic or crystallographic alignment, it is hard to give a clear conclusion based on the inconsistent experimental observation results [17, 27-29]. Figure 5a shows the distribution of GNBs is related to the FA. The statistical result shows that average angle between GNBs and FA is nearly $36.5^{\circ}$, and most of the angles' distribution is from $25^{\circ}$ to $50^{\circ}$ with a significant spread from $30^{\circ}$ to $45^{\circ}$. This result agrees with the previous studies about the angles' distribution during cold rolling at same strain $[6,7]$. GNBs are primarily parallel to the $\{111\}$ trace, with most misorientation angles being $<10^{\circ}$, as shown in Fig. 5b. These statistical results suggest that GNBs prefer a macroscopic and crystallographic alignment which is determined by grains' orientation.

Table 1 shows Schmid factors of all slip system in grains in Figs. 1, 2, and 3. The grains can be considered under plane strain during bending as the strain in transverse direction is relatively small. In Table 1 , Schmid factors for all 12 slip systems of all studied grains are calculated. The four of highest Schmid factor are in bold type, (ignore the sign of Schmid factor), as shown in Table 1 . About 93 in 130 grains form GNB microstructures during bending, and it is found that most GNBs coincide with the trace of $\{111\}$ slip planes. For example, Grain 1 in Fig. 1a, slip systems defined by the four highest Schmid factor are (111)[011] , (111)[101], $(11 \overline{1})[101]$, and (11) $)[\overline{1} 10]$. It is believed that these two coplanar slip systems form into two sets of GNB structures and parallel to the $\{111\}$ approximately [30]. For grain 6 in Fig. 3c, two coplanar slip systems with higher Schmid fac-

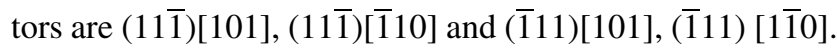
But only one set of GNBs parallel to (111) trace can be observed as Schmid factors in slip systems of (111)[101] and (111)[110] are similar. For grain 2 in Fig. 3c, the slip systems are defined by four highest Schmid factors, i.e.,

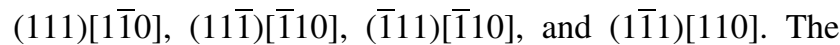
aligned dislocation boundaries do not exist in this grain due to the four Schmid factors are closely. These unique slip systems lead to the formation of equiaxial cells; hence, the less number of dislocation is observed in grains with Cube orientation during deformation [13].

\section{Conclusions}

The microstructures of 2060-T8 alloy during bending are studied with BSE, EBSD, and misorientation axes maps, and experimental results show that three types of microstructure are formed during bending.

The type of microstructure depends on the grain's orientation: Type A is mainly observed in grains with brass, copper, and Goss orientations which contain two sets of GNBs; type B is observed in grains with $\mathrm{S}$ orientation which contains one set of GNBs; type $\mathrm{C}$ is observed in grains with Cube orientation which consists of large dislocation cells.

Angle between GNBs and FA is in the range of $-45^{\circ}$ to $-30^{\circ}$ and $30^{\circ}$ to $45^{\circ}$, and most GNBs are approximately parallel to the trace of $\{111\}$ slip planes, which are identified by Schmid factor analysis.

Acknowledgments This work was financially supported by the Commercial Aircraft Corporation of China Ltd. The authors also thank Andrew Godfrey for the software of misorientation axes reconstructed maps.

\section{References}

[1] R.J. Rioja, J. Liu, Metall. Mater. Trans. A 43, 3325 (2012)

[2] Z.Q. Zheng, J.F. Li, Z.G. Cheng, Chin. J. Nonfer. Met. 10, 2337 (2011). (in Chinese)

[3] L.M. Karabin, G.H. Bray, R.J. Rioja, G. Venema, in ICAA13: 13th International Conference on Aluminum Alloys, (wiley, Pennsylvania, 2012)

[4] A. Davidkov, R.H. Petrov, P. De Smet, B. Schepers, L.A.I. Kestens, Mater. Sci. Eng. A 528, 7068 (2011)

[5] N. Hansen, Q. Liu, Scr. Metall. Mater. 32, 1289 (1995)

[6] Q. Liu, D.J. Jensen, N. Hansen, Acta Mater. 46, 5819 (1998)

[7] Z. Yao, G. Huang, A. Godfrey, W. Liu, Q. Liu, Metall. Mater. Trans. A 40, 1487 (2009)

[8] Q. Liu, X. Huang, D.J. Lloyd, N. Hansen, Acta Mater. 50, 3789 (2002)

[9] D.A. Hughes, N. Hansen, Acta Mater. 48, 2985 (2000) 
[10] X. Huang, N. Hansen, Scr. Mater. 37, 1 (1997)

[11] Q. Liu, Z.Y. Yao, A. Godfrey, W. Liu, Acta Metall. Sin. 45, 641 (2009). (in Chinese)

[12] X. Huang, G. Winther, Philos. Mag. 87, 5189 (2007)

[13] G. Winther, X. Huang, Philos. Mag. 87, 5215 (2007)

[14] N. Hansen, X. Huang, Acta Mater. 46, 1827 (1998)

[15] G.M. Le, A. Godfrey, C.S. Hong, X. Huang, G. Winther, Scr. Mater. 66, 359 (2012)

[16] B.L. Li, Dissertation, Tsinghua University, Beijing, 2003. (in Chinese)

[17] G. Winther, Acta Mater. 51, 417 (2003)

[18] P.J. Hurley, F.J. Humphreys, Acta Mater. 51, 1087 (2003)

[19] Q.L. Zhao, A. Godfrey, Chin. J. Stere. Image Anal. 15, 37 (2010). (in Chinese)

[20] Z.Y. Yao, Q. Liu, A. Godfrey, W. Liu, J. Chin. Electr. Microsc. Soc. 27, 452 (2008). (in Chinese)
[21] N. Hansen, C. Maurice, Q. Liu, Metall. Mater. Trans. A 29, 2333 (1998)

[22] N. Hansen, D.J. Jensen, Philos. Trans. R. Soc. A 357, 1447 (1999)

[23] N. Hansen, R.F. Mehl, Metall. Mater. Trans. A 32, 2917 (2001)

[24] K. Morii, H. Mecking, Y. Nakayama, Acta Metall. 33, 379 (1985)

[25] A. Godfrey, D.J. Jensen, N. Hansen, Acta Mater. 46, 823 (1998)

[26] A. Godfrey, D.J. Jensen, N. Hansen, Acta Mater. 46, 835 (1998)

[27] P.J. Hurley, P.S. Bate, F.J. Humphreys, Acta Mater. 51, 4737 (2003)

[28] G. Winther, X. Huang, A. Godfrey, N. Hansen, Acta Mater. 52, 4437 (2004)

[29] F.J. Humphreys, P.S. Bate, Acta Mater. 54, 817 (2006)

[30] Q. Liu, N. Hansen, Phys. Status Solidi A 149, 187 (1995) 